\title{
Due to intolerance of dental materials used for therapeutic treatment
}

\author{
Bukhara state medical Institute \\ O. A. Razhabov, Khayitova M. A., Usmonov N. U.
}

\begin{abstract}
Long-term clinical observations show that widely used dentures made of metals and their alloys are not indifferent, cause a number of diseases of the oral mucosa, and have social and medical consequences in the form of intolerance to dental prosthesis materials[1,2]. Currently, ceramic materials, plastics, stainless steels, cobaltchromium, silver-palladium alloys, gold-based alloys, platinum, etc. are used for orthopedic treatment. these alloys include the following metals: iron, chromium, Nickel, titanium, manganese, silicon, molybdenum, cobalt, palladium, zinc, silver, gold, etc. Signs of intolerance may occur when placing dentures and filling materials in the oral cavity. Intolerance to prosthetic materials can be caused by different reasons that have different mechanisms of pathology development. Solving problems with intolerance to dental materials in each patient requires analysis together with a dentist, an immunologist, and an immunological laboratory [1-4].
\end{abstract}

Keywords: orthopedic structures, local immunity

\section{Introduction}

The aim of the study was to study the nature of structural reorganization of the gingival mucosa and the dynamics of local oral immunity indicators during odontopreparation and tobacco Smoking in order to develop measures for the prevention of possible complications.

The purpose of this review is to examine the causes of intolerance to dental materials used for orthopedic treatment.

The reasons for the receipt of trace elements from dentures in saliva are wear of the metal surface, local corrosion and saliva chemistry. Stainless steel prostheses cause an increase in saliva of iron by 5.5 times, copper by 7 times, manganese by 1.7 times, and lead by 6 times. The content of trace elements in the saliva of individuals with intolerance to metal prostheses correlates with an increase in chromium by 6 times, and Nickel - by 3.4 times. The phenomena of galvanism that occurs as a result of electrochemical interaction in the presence of stainless steel dentures in the oral cavity account for 4 to $11 \%$ of cases. [7,8,9]. Also, intolerance to dental materials can be caused by an individual pathological reaction to the material. Another reason may be the poor hygiene of the oral cavity [9-12]. Oral galvanosis is a disease characterized by a number of non-specific clinical manifestations associated with chronic inflammation of the oral mucosa against the background of galvanic currents between dissimilar metals of dental prostheses [5-8].

\section{There are several symptoms of galvanosis:}

- The appearance of an unpleasant metallic and sour taste in the oral cavity.

The appearance of abnormalities of the taste buds with a violation of taste sensitivity.

- Manifestation of pathology of the salivary glands. Dryness in the oral cavity. It is caused by irritation of the nerve endings, which leads to abnormalities in the autonomic and Central nervous systems [13-15].

- Burning and itching sensations.

- Inflammatory process on the surface of the tongue.

There are also General physiological deviations from the norm: decreased vitality, complaints of head pain, nervous response to others [16-18].

Most often, these sensations begin to appear a month or two after orthopedic treatment, the material of which was stainless steel, or repeated prosthetics, the material of which was another material or alloy (clasp prosthesis made of chrome-cobalt alloy, gold compounds or others).

In places of fusion, an oxide film can be observed [20-23]. Signs of galvanosis develop very rarely and depend on the characteristics of the patient and the state of his oral cavity $[19,24]$. Very often people go to the dentist with such a problem as allergic reactions to dental prostheses. An allergic reaction is the body's response to environmental factors that can cause damage and inflammation of the mucous membranes [25].

\section{Results and discussions.}

Usually, an Allergy appears to metals that are used in the manufacture of prostheses: Nicole, cobalt, copper, chromium, as well as their combination. Such materials are used in the manufacture of cheap metal-ceramic structures of crowns and dental bridges, as well as as the basis for the production of removable dentures.

The most common signs of allergic reactions to metal: redness, itching and swelling of the mucous membranes in the oral cavity; irritation of the inner surface of the cheeks, tongue, lips; discomfort in the oral cavity of various types, such as dryness or excessive salivation, bitterness, sour taste, sore throat; exacerbation of bronchial asthma; rashes of various types in the oral cavity, on the skin around the lips, on the hands; fever; the most dangerous angioedema (Edema Quincke) [31-35]. I have studied local immunological indicators of the oral cavity, which play an important role in providing local protection. By changing these indicators, it is possible to judge the 
presence of inflammation in the process of adaptation to the orthopedic structure, as well as the effect on the oral tissue of the material from which the structure is made, i.e., the presence of an allergic reaction or the phenomenon of galvanism.Recently, due to the widespread use of plastics to replace metal, the problem of plastic intolerance has come to the fore.

Allergies can appear not only on metals. However, plastics that consist of monomers can sometimes cause an allergic reaction. It is worth noting that now nylon prostheses or Quadrotti are produced, which are made of soft plastics, they do not pose a danger to humans [26, 27, 35].

Causes of plastic intolerance: mechanical trauma to the oral mucosa with a prosthesis; allergic and toxic-chemical effects on the oral mucosa of substances that are part of prosthetic plastics; non-physiological conditions under a removable prosthesis; effects on the oral mucosa of plaque components on the prosthesis; somatic diseases, anemia, diabetes, hormonal disorders; iatrogenic or psychogenic factors [28, 29].

The main pathogenetic factors for the development of galvanosis are galvanic currents, metal corrosion, and pathological changes in saliva [5]. In this regard, when choosing structural materials for dental prostheses, many researchers analyze the corrosion resistance of various metal alloys in artificial saliva, study metal changes in the patient's oral cavity, but the issues of their clinical compatibility are not fully investigated.

In studies A. A. Timofeev presented quite convincingly that the diagnosis of "Galvanos the mouth" applies in the presence of characteristic clinical symptoms: metallic taste, bitter taste, burning tongue and sour-salty taste, change in salivation, the sensation of current flow through the tooth, deterioration of the General condition.

In this regard, much attention is paid to the clinical and theoretical aspects of the problem of allergic reactions in domestic and foreign medical science, including dentistry.

\section{Conclusions}

We analyzed data on indicators of local immunity of the oral cavity, which are an important criterion for evaluating the quality of treatment and serve as one of the methods for preventing errors and complications in prosthetics with orthopedic structures.Analysis of the literature used suggests the need for further study of the diagnosis, clinical manifestations and prevention of galvanosis in patients. Aspects of evaluating the compatibility of dental alloys used in orthopedic structures need to be clarified. Currently, specialists do not have a single comprehensive approach to the diagnosis and prevention of galvanosis in dental patients.

\section{REFERENCES}

1. V abadjyan. n. Changes in microcirculation parameters of the prosthetic bed mucosa after prosthetics with removable prostheses // Methods for studying regional blood circulation and microcirculation in the clinic.- Saint Petersburg, 2003. - P. 113-114.

2. Arutyunov S. D. and Lebedenko. Yu. Maksimovsky Yu. M. Galvanic processes in stamatology. 2012$172 \mathrm{c}$.

3. Arutyunyan A.V., Vanyan N. G. On the study of galvanism in the oral cavity // Bulletin of dentistry, Yerevan, 2007, Vol. 4, no. 2. - p. 56-57.

4. Bazhanov N. N., V Kozlov.A., T Robustova.G., Maksimovsky Yu. M. State and prospects of prevention and treatment of purulent-inflammatory diseases of the maxillofacial region // Dentistry, 1997, 2. - gr. 1519 ,

5. Banchenko G. V. Fleischer G. M. et al. Eletromagnetic Allergy galvanosis medical alphabet / / dentistry, 2/2012. - P. 42-51.

6. Benkovskaya S. G., Rastorguev B. T. Comparative evaluation of periodontal tissues in patients with metal and cermet prostheses // New in dentistry, 1996; 3. - gr. 21-22.

7. Budypina S. M., Dechtyarev V. P. Physiology of the maxillofacial region / / Moscow, Meditsina, 2001. $350 \mathrm{~S}$.

8. Byakova Zh. s. Assessment of regional blood flow when using dental prostheses // Nizhegor. Med. Zhurn., 2003. - pp. 164-165.

9. Vanyan N. G., Tatintsyan L. V., Yesayan L. K., Shaginyan A. G., Avetisyan A. A. Influence of nonremovable metal prostheses in the oral cavity on the state of teeth and periodontal tissues // Medicine, science and education, Yerevan 2009/2, pp. 78-81.

10. Vanyan N. G. Results of studies of the state of the oral cavity in patients who wear bridges for a long time // Bulletin of dentistry, Yerevan, 2011, Vol. 8, no. 3. - p. 6-8.

11. Volozhin A. I. Allergy and other types of intolerance in dentistry, etiology, pathogenesis, principles of treatment / / method. a manual for students of Stom.faculty. M. MMSI, 1994. - p. 89.

12. Volyntsev V. M. Clinical and laboratory indicators of intolerance to metal alloys // Abstract. Diss. candidate of medical Sciences. Kiev, 1996. - p. 17.

13. Gozhy A. G. Clinical manifestations of electrochemical processes caused by finishing treatment of stainless steel dentures/ A. G. Gozhy, G. R. Sagatelyan, L. D. Gozhaya // Dentistry. - 1998. - N3. - P. 4650 . 
14. Razhabov O. A. Clinical changes in the oral cavity when using metal-ceramic dentures/ / Zh. Tibetaanse kun 1(29)2020 p. 322-325.

15. Zufarov A. S., Israilov, I. H., X Rakhmanov.Sh. Morphological evaluation of prosthetic bed after orthopedic intervention by low-intensity laser radiation // Proc.scientific.Tr. Kazan, 1995.- KN.1.-gr.134136.

16. Kapitonova Yu. M. Treatment of secondary adentia with bridge-like prostheses / / author's thesis of the candidate of medical Sciences, Moscow, 2002.

17. Knyazeva M. B. Preparation of teeth and periodontium for the use of metal-ceramic prostheses // Abstract. dis. Cand. med. Sciences.

18. Kozlov V. I., Gurova O. A., Azizov G. A. and others. Laser Doppler Doppler flowmetry in assessing the state of blood microcirculation / / methods for studying regional blood circulation and microcirculation in the clinic. Saint Petersburg, 2004, pp. 71-72.

19. Kolesova T. V. Analysis of the results of orthopedic treatment with solid-cast metal-ceramic dentures / / author's abstract of the Cand. med. sci. Volgograd, 1999. - p. 294.

20. raigrodski AJ. Modern materials and technologies for all-ceramic fixed partial dentures: literature review / / J Prosthet Dent 2004; 92. - Pp. 557-62.

21. Radzhabov O. A. clinical and functional changes in the oral cavity when using metal-ceramic dental prostheses/ / South Asian academic scientific journals Volume 10 issue 2, February 2020g. 209-215.

22. Schillinburg H., Jacobi R., Brackett 5. Fundamentals of dental preparation for cast metal and porcelain restorations / / Quintessence Publishing Co. Chicago-London-Berlin-Sao Paulo-Tokyo and Hong Kong Sec Print 1991. - p. 390.

23. Sorensen J. Lava system for CAD / / Cam production of high-strength precision fixed dentures. quintessence dent technol 2003; 26:57-67.

24. Kopye FM. Practice without metal: a myth? Reality? The desired goal? // J Esthet Restor Dent 2001; 13:59-67.

25. Is located j, Nutt g, Mautsch W, Spickerman h, Anusavice kJ. Maximum fit of fixed partial dentures based on aluminum oxide and zirconium, made using CAD / / CAM. Oper Dent 2001; 26. - Pp. 367-74. 